\title{
Comparison of primary human hepatocytes and HepG2 cells as models to study the development of hepatic steatosis
}

\author{
Z.J. Huggett ${ }^{1}$, J.M. Brameld, A.J. Bennett ${ }^{2}$ and A.M. Salter ${ }^{1}$ \\ ${ }^{1}$ Division of Nutritional Sciences, School of Biosciences, University of Nottingham, Sutton Bonington Campus, LE12 \\ $5 R D$ and \\ ${ }^{2}$ School of Life Sciences, University of Nottingham, Queens Medical Centre, NG7 2UH
}

Non-alcoholic fatty liver disease (NAFLD) is the hepatic consequence of the metabolic syndrome. It is highly prevalent in Western populations and puts a huge economic burden on health services ${ }^{(1)}$. NAFLD begins with the hepatic accumulation of triacylglycerol in lipid droplets within the hepatocytes (steatosis). A greater understanding of the impact of specific nutrients on NAFLD development and progression is needed to help identify better dietary recommendations, treatments and prevention strategies. This study aimed to determine whether HepG2 cells, a commonly used human hepatoma cell line, respond to nutritional stimuli in a similar manner to primary human hepatocytes $(\mathrm{PHH})$ in regards to lipid accumulation.

PHH were obtained from 3 patients undergoing elective liver surgery and were obtained with full ethical approval and informed consent. HepG2 cells or PHH seeded in collagen coated 96-well plates were treated with glucose $(5 \mathrm{mM}$ or $11 \mathrm{mM})$, fructose $(0 \mathrm{mM}, 2 \mathrm{mM}$ or $8 \mathrm{mM})$, fatty acids $(0 \mu \mathrm{M}$ or $200 \mu \mathrm{M}$ of a mixture of palmitic, oleic and linoleic acids in the ratio $2: 2: 1)$ or vehicle controls for 48 hours before determining relative intracellular lipid content using a Nile Red ${ }^{(2)}$ staining assay. Cells were then lysed by freezing and the DNA content measured to normalise for cell number. Data was analysed using three-way ANOVA.

HepG2 cells only accumulated lipid in response to treatment with $200 \mu \mathrm{M}$ fatty acids $(P<0.0001)$ whereas PHH accumulated lipid in response to fatty acids $(P<0.0001)$ and fructose $(P<0.0001)$ (Figure 1$)$. Fatty acid $\times$ glucose and fructose $\times$ fatty acid interactions were suggested in $\mathrm{PHH}(P=0.057$ and $P=0.076$ respectively) and were significant in some individual livers.
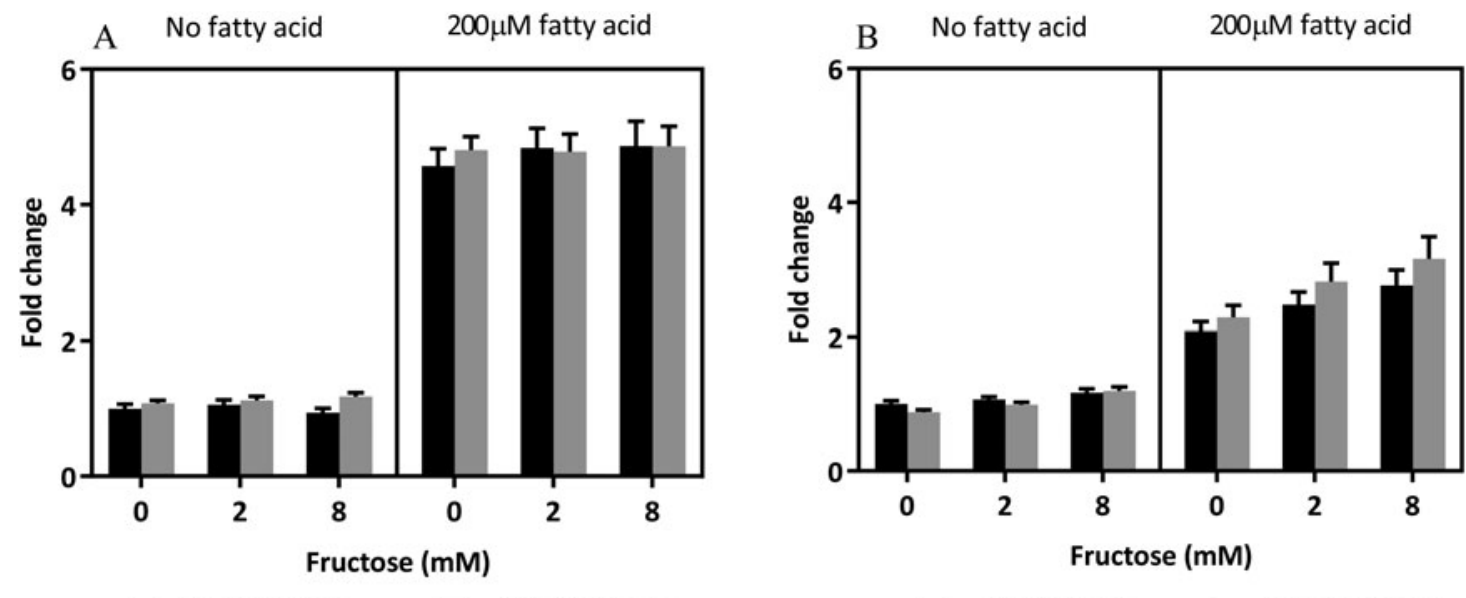

$5 \mathrm{mM}$ glucose $\quad 11 \mathrm{mM}$ glucose

$5 \mathrm{mM}$ glucose

$11 \mathrm{mM}$ glucose

Fig. 1. Nutrient effects on intracellular lipid content (A) HepG2 cells and (B) primary human hepatocytes. Lipid was measured by Nile Red staining after 48 hours of treatment and normalised to DNA content. Fold change relative to $5 \mathrm{mM}$ gluc $+0 \mathrm{mM}$ fruc $+0 \mu \mathrm{M}$ fatty acid. Values are expressed as mean + SEM. A: $n=20$ (4 plates each with $n=5), B: n=30$ (3 livers each with 2 plates with $n=5$ replicates)

In conclusion, HepG2 cells and primary human hepatocytes respond differently to glucose and fructose treatment in terms of lipid accumulation. The only consistent effect was increased intracellular lipid with fatty acid treatment, with a greater magnitude in HepG2 cells. Further investigation into genetic factors, gene regulation and substrate utilisation will help to elucidate the mechanisms behind these differences. HepG2 cells may not be a physiologically relevant model for studying NAFLD.

This work was funded by The University of Nottingham and The Rank Prize Funds.

1. Younossi ZM, Blissett D et al. (2016) Hepatology 64, 1577-1586

2. Greenspan P, Mayer EP et al. (1985) J Cell Biol 100, 965-973 